\title{
A multifaceted intervention to reduce inappropriate polypharmacy in primary care: research co-creation opportunities in a pilot study
}

A goal of primary care research is to inform and facilitate beneficial change in health care delivery for both patients and clinicians in a way that is efficient for providers and economically sustainable for the health care system. Co-design or co-creation is a process whereby researchers and stakeholders jointly contribute to the ideation, planning, implementation and evaluation of new services and systems as a possible means to optimise the impact of research findings. ${ }^{1,2}$ Co-creation represents the highest form of stakeholder engagement, building on existing theories such as community-based participatory research, ${ }^{3}$ and emphasises the creation of value for both end users and researchers.

However, it is often infeasible to co-create with all stakeholders at all stages of a research project. Decisions must be made about which stakeholders to involve at different stages to achieve the greatest return on investment for researchers' and stakeholders' time and contributions, considering the context in which change is likely to occur.

In designing a controlled pilot study of a multifaceted intervention to reduce inappropriate polypharmacy in primary care involving 20 general practitioners and more than 150 patients in south-east Queensland, we identified both patients and prescribers as the most important stakeholders. For this project, however, we proposed that the critical gateway to effective co-creation in the first instance was the GP, with both the GP and patient to be involved in evaluating the pilot to inform future development. In this article, we describe the rationale for this approach, the process, challenge and value of cocreating with GPs in the planning and implementation of the intervention, and the anticipated value of involving both patients and GPs in the pilot's evaluation.

\section{Background}

The impetus for this co-creation research project was Australian data suggesting high rates of potentially inappropriate medication use among older Australians, and its association with significant patient harm. ${ }^{4}$ The aim of the project was to design and pilot a multifaceted deprescribing intervention to minimise inappropriate polypharmacy in older people in primary care.

Deprescribing is the systematic process of identifying and discontinuing the use of medicines where the actual or potential harms outweigh the benefits, giving due consideration to an individual patient's care goals, current level of functioning, life expectancy, values and

\section{Summary}

- Co-creation (or co-design) represents the highest form of stakeholder engagement, but it can be infeasible to co-create with all stakeholders through all stages of a research project.

- The choice of stakeholders for co-design will depend on the study purpose and context of change.

- For this deprescribing pilot study, general practitioners were recognised as a critical gateway for co-creation, with patients' perspectives of the deprescribing process to be assessed in the evaluation of the pilot.

preferences. ${ }^{5}$ Box 1 shows an overview of the decision algorithm for use by the GPs in this pilot study. Deprescribing is an inherent principle of quality use of medicines and is part of good prescribing. ${ }^{7}$ The study presented here represents early-phase piloting and developmental work, as described by the United Kingdom Medical Research Council's guidance for complex interventions. ${ }^{8}$

\section{Rationale for co-creation with GPs}

This deprescribing pilot study, as a quality prescribing initiative, aimed to effect change at the microsystem level (ie, the provider-patient interaction). Relevant stakeholders for co-design could have included patients (and family or carers if relevant), GPs, medical specialists, hospital providers, community pharmacists and other members of the primary health care team.

Michele M Foster PhD, BSocWk(Hons) $)^{1,4,}$

Christopher R Freeman PhD, AdvPracPharm, BCACP ${ }^{2,}$

Ian A Scott FRACP, MHA, MEd ${ }^{1,2,5}$

1 Centre of Research Excellence in Quality and Safety in Integrated Primary-Secondary Care, Brisbane, QLD.

2 University of Queensland, Brisbane, QLD.

3 Charming Institute, Brisbane, QLD.

4 Menzies Health Institute Queensland, Griffith University,

Gold Coast, QLD. 5 Princess Alexandra Hospital, Brisbane, QLD.

k.anderson8@uq.edu.au doi: 10.5694/mjal6.00125
There were several reasons for prioritising GPs for codesign in this pilot. Recent literature exploring patients' perspectives on deprescribing indicated that their GPs can be highly influential in encouraging patients to cease taking inappropriate medicines, ${ }^{9}$ confirming that GPs exert considerable influence on what is discussed during consultation time with patients. ${ }^{10}$ Deprescribing is a highly nuanced, individualised process that requires comprehensive, holistic review and follow-up of a patient. Accordingly, the GPs' often long term and trusted relationship with, and tacit knowledge of, their patients place them in an ideal position to engage patients in the deprescribing process and participate in decisions about the continuation or discontinuation of long term therapy. ${ }^{11}$ Giving due consideration to the organisational structure and operation of primary care, and the asymmetry of medical knowledge between patients and GPs, the GP was considered to be best positioned to raise the issue of deprescribing, and collaborate with patients 
1 Abridged deprescribing algorithm ${ }^{5,6}$

- Ascertain all medicines that the patient is taking and the reasons for using each one.

- Consider the overall risk of medicine-induced harm in the individual patient, determining the required intensity of the deprescribing intervention.

- Assess each medicine for its eligibility to be discontinued, and deprescribe medicines:

- without a clear or valid indication;

- that are part of a prescribing cascade;

- where the actual or potential harms clearly outweigh any benefits;

- for symptom control if they are ineffective or if symptoms have resolved;

- that are preventive in nature, and that are unlikely to confer any important benefit in a patient's remaining life span; and

- that are imposing an unacceptable treatment burden.

- Establish an order of priority for discontinuing medicines.

- Implement and monitor the medication discontinuation regimen.

Adapted from Scott and colleagues. ${ }^{5}$

and other highly influential stakeholders such as specialists and health professionals, to facilitate the process as appropriate. We were also aware of research identifying patient attitudes and barriers to deprescribing initiatives, which we used to inform our study design., ${ }^{9,12}$ For all these reasons, we decided to pursue co-design with GPs in the first instance. Although co-design would not be undertaken with the patients at the front end, the pilot evaluation would include interviews with both patients and GPs aimed at ascertaining the impact and acceptability of the deprescribing process.

\section{The challenge and reward of research co-creation in primary care}

GPs are a busy and heterogeneous group of health professionals, whose practice settings vary markedly in terms of administrative and clinical support, resourcing and infrastructure, capacity and readiness for change. ${ }^{13}$ Engagement of GPs in co-creation research must occur at times and places that are optimally convenient for them, and must recognise the immense variability among GPs and their work contexts. The following describes examples of how GPs were involved in the planning, implementation and evaluation of the project in ways that recognise their busyness and diversity.

\section{Planning}

A collective approach to co-creating new systems or services is often preceded by information gathering and conceptual ideas. ${ }^{1}$ We undertook a systematic review of the literature of prescribers' barriers and enablers to minimising the use of potentially inappropriate medications and used its findings to inform the first stage of co-creation. ${ }^{14}$ We also undertook focus group discussions with GPs from five large practices in the designated pilot study catchment area with two objectives: (i) to identify local barriers and enablers to deprescribing, as local context is highly influential in shaping clinician behaviour, ${ }^{8}$ and compare these with the findings of the review; and (ii) to determine the perceived applicability and utility of a purposively designed deprescribing framework in routine care, modified from one shown to be an effective tool for deprescribing in hospitals. ${ }^{15}$

The findings of the focus group discussions and the literature review together with liaison with a senior academic GP adviser experienced in the care of older multimorbid patients, were critical in understanding the context in which the intervention would be applied and consequently, in formulating its key components, including:

- conducting an interactive training workshop for GPs on deprescribing, based on the deprescribing framework;

- identifying patients at high risk of medication misadventure;

- scheduling extended deprescribing appointments with GPs for their high risk patients (with follow-up and referral at the GP's discretion); and

- providing the option of referring patients to an accredited pharmacist trained in deprescribing to review a patient's medicines to help GPs overcome some of their barriers to deprescribing, such as limited time.

GPs, with their patients, would be free to collaborate with or involve other health professionals throughout the deprescribing process, in recognition of the heterogeneity of GP work environments, care teams and with respect for the GPs' professional autonomy.

\section{Implementation}

The next step was working with GPs recruited into the pilot study to co-design practical tools that would help meet study objectives. ${ }^{1}$ The following describes two examples, wherein a flexible, iterative research design was used that could respond to the needs of different general practices.

Example 1: Identifying patients at high risk of medication misadventure. The research team worked closely with the principals (or their delegates) of the practices recruited to the pilot to develop a standardised but customisable patient management software query that would help identify patients at high risk according to evidence-based criteria. This involved the use of the same medical software (Best Practice Software) at all but one site. A comparable query was performed using an external reporting software tool at the site that used different software. To account for variations in data quality across the sites, the software query was combined with a 
documented manual screening process so that each GP could generate a sample of consecutive eligible patients for study recruitment. The co-design of the software query minimised the extent of manual screening required by GPs and practice staff, but maintained a degree of standardisation of non-negotiable search criteria important to the research team across all of the sites.

Example 2: Co-designing a multipurpose tool for use during the deprescribing appointment. Focus group discussions with GPs emphasised that any tool for deprescribing should ideally be integrated into the medical software used in general practice. The research team aimed to minimise the burden of documentation for GPs as part of the project.

A tool or template, easily imported into the electronic consult notes, was developed and served three functions: collecting data for the research team; acting as a memory prompt for the GPs of the key steps of deprescribing; and providing a framework for documenting the

deprescribing consultation. The draft tool was designed by the team, with the aid of a practising GP with research and software experience, after considering GPs' work processes and the required sequential steps of the deprescribing framework.

This template (Box 2) was subsequently trialled in the medical software used by participating GPs who

\section{Template for use by GPs in deprescribing consultations*}

Patient attended for appointment as part of University of Queensland study.

Medication history taken and list reconciled? Y or N? If no, why?

Each medication reviewed for utility? Y or N? If no, why?

Medications eligible for deprescribing -

- Medication, coded reason for ceasing or restarting (see below), GP recommended plan, plan after discussion with patient

This step is repeated for as many medications as deemed appropriate.

Next appointment to be scheduled <insert date>

- Coded reasons for CEASING medications (free text in consult notes OR select from drop down list) -

1. No clear or valid indication (including contraindication)

2. Prescribing cascade

3. Actual or potential harms $>$ benefits

4. Symptom control

a. Ineffective

b. Symptoms resolved

5. Time until benefit questionable

6. Unacceptable treatment burden

7. Patient stopped it

- Coded reasons for RESTARTING medications previously deprescribed (free text in consult notes) -

1. Restart - Symptom relapse

2. Restart - Withdrawal syndrome

* A letter template for completion and forwarding to lead researcher in regard to any adverse events thought probably or possibly related to deprescribing was also implemented. attended the preparatory deprescribing workshop. This resulted in additional decision support being added to the tool on the basis of suggestions made by GPs. All participants recognised that having a multipurpose tool integrated into the medical software increased the chance of it being used in routine care and capturing all information critical to project evaluation.

\section{Evaluation}

The future evaluation of the deprescribing project will involve a mixed-methods approach. The primary outcome measure is a change in the total number of regular medications taken by older people at high risk of medication misadventure. Secondary measures will include change in the number of medications taken as needed (prn), the number of medications to have doses changed, drug classes commonly deprescribed and process measures such as the proportion of patients attending their deprescribing appointment. A formal process for ascertaining and recording any instance of actual or potential patient harm arising from deprescribing has been implemented. Changes in patient attitudes towards deprescribing and quality of life will be assessed by pre- and post-questionnaire surveys. Interviews with GPs and patients will explore the acceptability and impact of the deprescribing process. The results of this pilot study will help determine if there is sufficient justification for seeking funding for a larger-scale trial that evaluates the deprescribing intervention used, or an amended version of it, across a wider spectrum of general practice settings.

\section{Conclusion}

Utilising a co-creation approach in research is both challenging and rewarding. The exact approach that is taken needs to be tailored according to the study purpose, views of key participants and context of change. The level of engagement between researchers and end users will vary at different stages of planning and implementation to ensure maximum value for both parties.

Competing interests: Kristen Anderson and Christopher Freeman are accredited pharmacists who receive payment for medication review services through the Sixth Community Pharmacy Agreement. Kristen Anderson, Michele Foster and lan Scott are funded through a National Health and Medical Research Council grant under the Centre of Research Excellence in Quality and Safety in Integrated Primary-Secondary Care (grant ID, GNT1001157)

(c) 2016 AMPCo Pty Ltd. Produced with Elsevier B.V. All rights reserved.

1 Sanders EBN, Stappers PJ. Co-creation and the new landscapes of design. CoDesign 2008; 4: 5-18.

2 Jackson CL, Greenhalgh T. Co-creation: a new approach to optimising research impact? Med J Aust 2015; 203: 283-284. https://www.mja.com.au/ journal/2015/203/7/co-creation-new-approach-optimising-research-impact

3 Viswanathan M, Ammerman A, Eng E, et al. Community-based participatory research: assessing the evidence. Evid Rep Technol Assess (Summ) 2004; 99: 1-8. http://www.ncbi.nlm.nih.gov/books/NBK11852/ (accessed Feb 2016).

4 Scott IA, Anderson K, Freeman CR, Stowasser DA. First do no harm: a real need to deprescribe in older patients. Med J Aust 2014; 201: 390-392. 
https://www.mja.com.au/journal/2014/201/7/first-do-no-harm-real-needdeprescribe-older-patients

5 Scott IA, Hilmer SN, Reeve E, et al. Reducing inappropriate polypharmacy: the process of deprescribing. JAMA Intern Med 2015; 175: 827-834.

6 Anderson K, Freeman C, Rowett D, et al. Polypharmacy, depresribing and shared decision-making in primary care: the role of the accredited pharmacist. J Pharm Pract Res 2015; 45: 446-449.

7 Australian Government Department of Health and Ageing. National medicines policy, 2000. http://www.health.gov.au/internet/main/publishing. nsf/Content/B2FFBF72029EEAC8CA257BF0001BAF3F/\$File/NMP2000.pdf (accessed Feb 2016).

8 Craig P, Dieppe P, Macintyre S, et al. Developing and evaluating complex interventions: the new Medical Research Council guidance. BMJ 2008; 337: al655.

9 Reeve E, To J, Hendrix I, et al. Patient barriers to and enablers of deprescribing: a systematic review. Drugs Aging 2013: 1-15.
10 Greenhalgh T, Snow R, Ryan S, et al. Six "biases" against patients and carers in evidence-based medicine. BMC Med 2015; 13: 1-11.

11 Royal Australian College of General Practitioners. Becoming a GP in Australia. What is a GP? http://www.racgp.org.au/becomingagp/whatis-a-gp/ (accessed Dec 2015).

12 Reeve E, Wiese MD, Hendrix I, et al. People's attitudes, beliefs, and experiences regarding polypharmacy and willingness to deprescribe. J Am Geriatr Soc 2013; 61: 1508-1514.

13 McMullen H, Griffiths C, Leber W, Greenhalgh T. Explaining high and low performers in complex intervention trials: a new model based on diffusion of innovations theory. Trials 2015; 16: 242.

14 Anderson K, Stowasser D, Freeman C, Scott I. Prescriber barriers and enablers to minimising potentially inappropriate medications in adults: a systematic review and thematic synthesis. BMJ Open 2014; 4: e006544.

15 McKean M, Pillans P, Scott IA. A medication review and deprescribing method for hospitalised older patients receiving multiple medications. Intern Med J 2016; 46: 35-42. 